\title{
Intersection line Length Normalization in CT Projection Data
}

\author{
Jan Müller, Thorsten M. Buzug \\ Institute of Medical Engineering, University of Lübeck, 23538 Lübeck, Germany \\ mueller@imt.uni-luebeck.de
}

\begin{abstract}
We present a method to improve the quality of common sinogram restoration algorithms, which are used for metal-artifact reduction in X-ray CT. The presented approach is based on the idea that the intersection length of all beams through the object of interest can approximately be calculated from a preliminary reconstruction of the object. Incorporating this information to the reconstruction allows significant reduction of streak artifacts. Additionally, it is shown that the information about intersection length may also be used to improve the detection of metal shadows within the sinogram.
\end{abstract}

\section{Introduction}

As shown on the left side in Fig. 1, metal objects inside the field of view cause artifacts in the reconstructed CT image. The predominant effect for these artifacts arises from inconsistent sinogram entries, which are a direct consequence of non-linear beam-hardening. One approach to correct for these erroneous values and, therefore, to reduce metal artifacts is to replace all corrupted sinogram entries with artificially generated values. If these surrogate values are chosen for instance by incorporating a priori knowledge, the quality of the reconstructed image is improved. There are several ways to calculate these artificial values. Two methods, which are related to each other are presented in [1] and [2]. A chain of clustering, segmentation and forward/backward projection is used to reconstruct a preliminary image, which represents an approximate distribution of air, soft tissue, and bones. A forward projection of this image is then used to fill the gaps in the original sinogram. A second approach is presented in [3] and [4]. Here, corrupted data is replaced with interpolated values from adjacent detectors. This interpolation is usually done independently for each view. In [5] a method is proposed, which uses data from more than one view to generate new values by means of an directional interpolation scheme.

In the present paper a method is proposed that can generally be incorporated into any interpolation based method. It is shown that a normalization of the sinogram improves both the reduction of metal artifacts and the detection of corrupted data compared to interpolation methods based on the original projection data. 
Fig. 1. The uncorrected reconstruction $f$ (left) and the image support $\operatorname{supp}(f)$ (right), which is generated from an image which was corrected with a common metal artifact reduction method (Fig. 4)

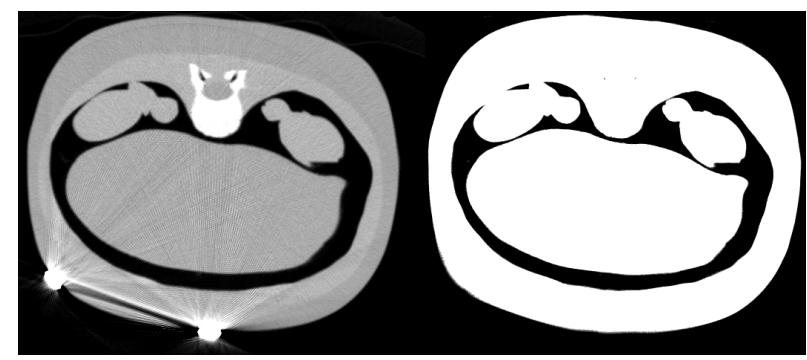

\section{Methods}

A problem which complicates the interpolation (and also the actual detection of corrupted sinogram entries) is that the value of a particular sinogram entry does not only depend on the material of the object but also on the intersection line length of the corresponding beam through the object: For example, a beam attenuated by a metal object and having a short intersection line with the body may have a smaller attenuation value than a beam, which is only attenuated by soft tissue and bone, but having a long intersection line through the body.

To reduce this influence, a normalization of the sinogram with respect to the intersection line lengths of each beam is proposed. It is based on the idea that the shape of an object can be approximately reconstructed even if the projection data is corrupted by the presence of metal objects. The calculation of the normalized data and its application to metal artifact reduction are as follows:

1. Creation a binary image which separates air and tissue (Fig. 1)

2. Calculation of a forward projection of the image. The values of this data are proportional to the intersection line length of each beam with the object (Fig. 2, middle image)

3. Division of the original sinogram data by the intersection length information. This results in a normalized sinogram, which contains the average attenuation value for each beam (Fig. 2, right image)

4. Detection of metal shadows and/or application interpolation based metal artifact reduction methods

5. Multiplication of the corrected normalized sinogram by the length-sinogram

6. Reconstruction of the image

Since metal artifacts in the uncorrected reconstruction may complicate the binarization process, it is recommend to apply a simple sinogram interpolation scheme as presented in [3] and [4]. Although this preliminary reconstruction still contains artifacts (Fig. 4, top row), it is nevertheless possible to extract shape information with a simple thresholding segmentation. The division in step 3 must 
Fig. 2. The original sinogram (left), the forward projection of the mask (center) and the normalized sinogram (right)

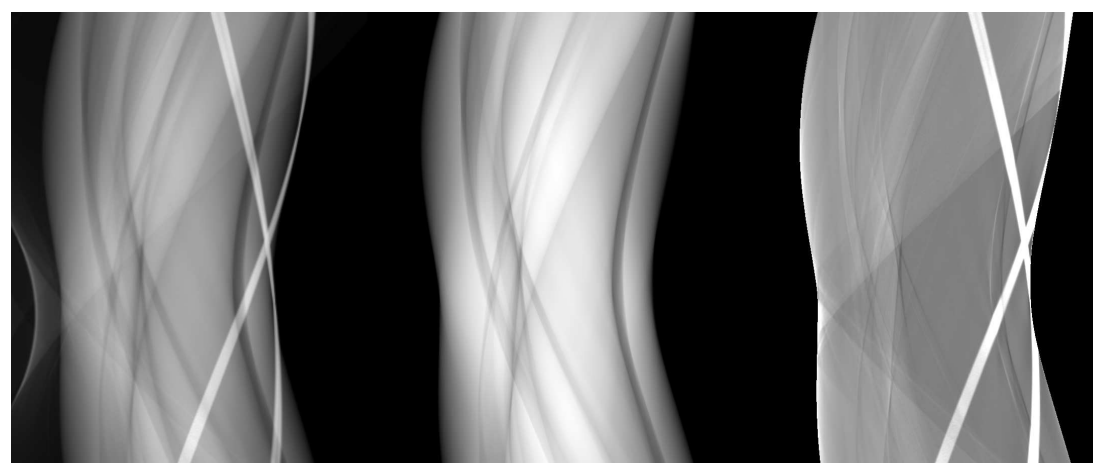

be regularized in order to avoid divisions by very small numbers or zero at the edge of the object.

To demonstrate the influence of the normalization a scan of an abdominal CTphantom with two externally attached steel markers is used. The corresponding sinogram is shown in Fig. 2. The reconstructed cross section including the metal artifacts can be seen in Fig. 1 and Fig. 4 respectively. The sinogram was restored using the linear and spline based methods as described in [3] and [4]. Additionally, the same interpolation methods were both applied to the normalized sinogram.

\section{Results}

All three sinogram types are presented in Fig. 2. The profile of one view from the original and the normalized sinogram are plotted in Fig. 3. As it can be seen, the overall appearance of the averaged sinogram is flatter than the original one. Beams through metal objects are more prominent, because of their larger normalized attenuation. This information can be used for more advanced detection schemes of metal shadows. However, the present paper deals only with the improvement of the performance of the interpolation.
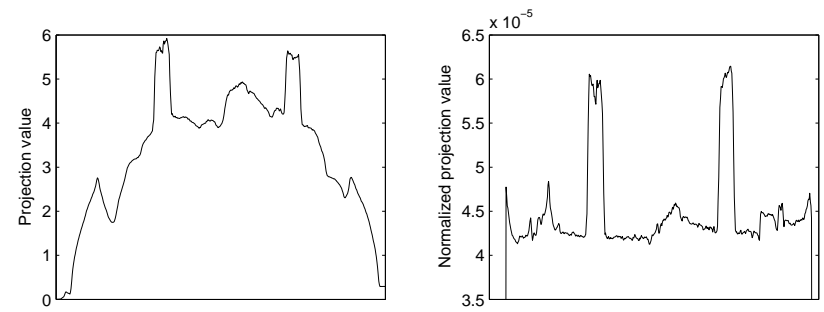

Fig. 3. The same view for both the original and the normalized sinogram 
Fig. 4. Upper row: the uncorrected reconstruction of the phantom with (left) and without (right) markers. The second image serves as ground truth. Middle row: traditional linear (left) and spline (right) interpolation. Bottom row: the same interpolation methods, but this time applied to normalized sinogram data
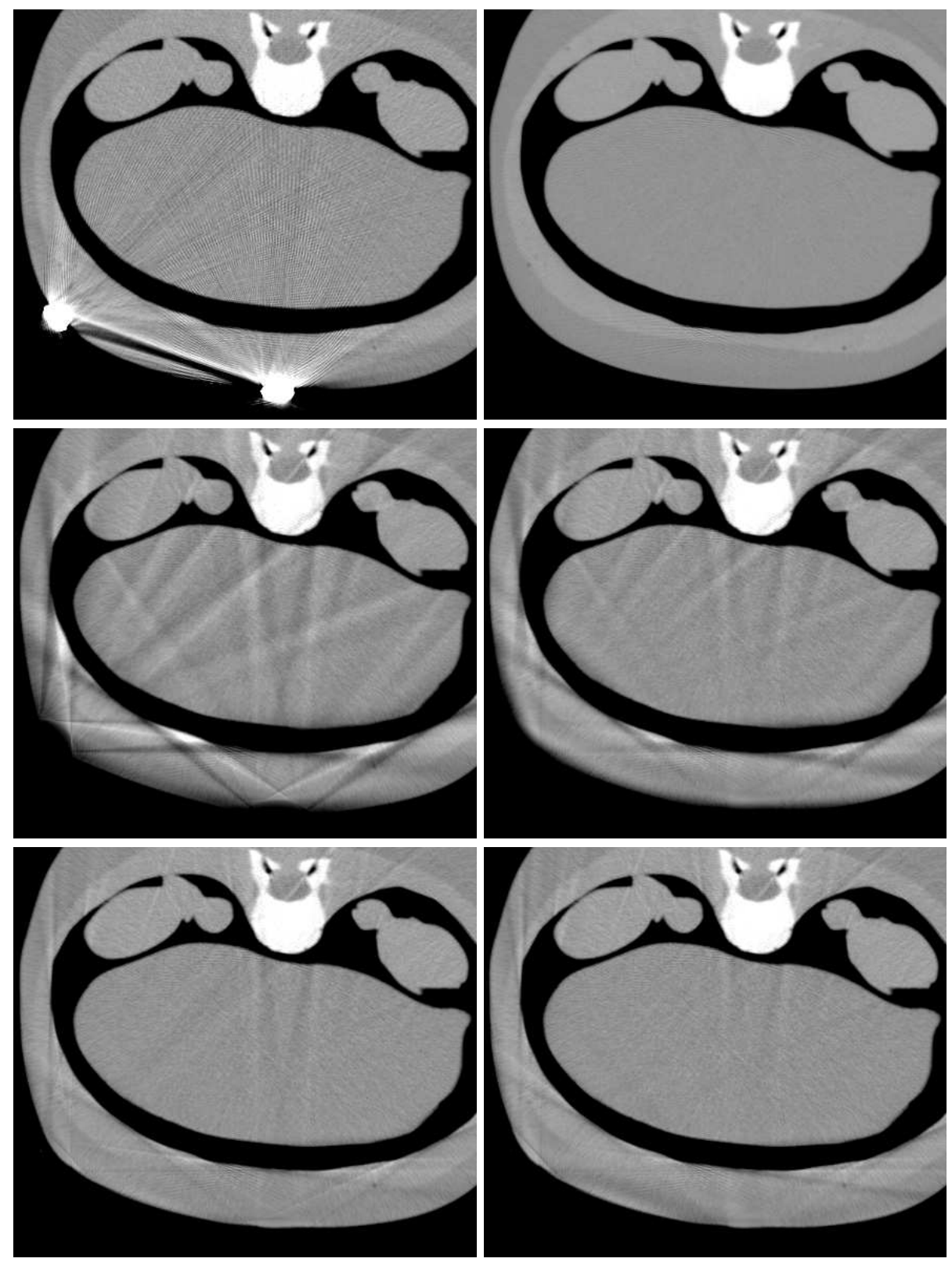
Fig. 5. Top row: Projection data with (left) and without (right) metal markers. The second scan serves as ground truth. Bottom row: Restorated sinograms using linear interpolation on original (left) and normalized (right) data.
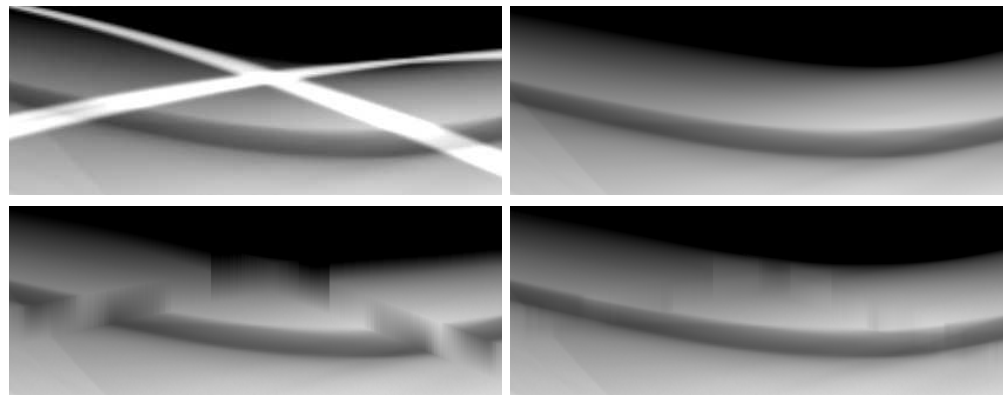

In the lower row of Fig. 5, results of the linear interpolation method applied to original (left) and normalized (right) data are shown. The interpolation result using normalized data resembles the ground truth data (upper right image) more closely, because the sinusoidal traces in the sinogram propagate into the interpolated areas in a natural way. The reconstruction results for linear (left) and spline based (right) interpolation methods applied to both type of datasets are shown in Fig. 4. As it can be seen, the use of normalized data significantly reduces streak artifacts which are characteristic for common interpolation based sinogram restoration methods.

\section{Discussion}

The results presented in Fig. 5 show that the combination of normalized sinogram data and simple interpolation schemes leads to a more plausible interpolation and, therefore, to a considerable reduction of interpolation artifacts (Fig. 4). The reason for this is, that at least an approximate calculation of the intersection length is possible for all beams. Thus, some information of the discarded projection data is still known and can be used to improve the interpolation.

\section{References}

1. Bal M, Spies L. Metal artifact reduction in CT using tissue-class modeling and adaptive prefiltering. Med Phys. 2006;33(8):2852-9.

2. Schmitt H, Timmer J, Koehler T. A three-pass method for metal artifact reduction in computed tomography. Med Phys. 2007;34:2571.

3. Glover GH, Pelc NJ. An algorithm for the reduction of metal clip artifacts in CT reconstructions. Med Phys. 1981;8(6):799-807.

4. Kalender WA, Hebel R, Ebersberger J. Reduction of CT artifacts caused by metallic implants. Radiology. 1987;164(2):576-7.

5. Oehler M, Buzug TM. Statistical image reconstruction for inconsistent CT projection data. Methods Inf Med. 2007;3:261-9. 